\title{
LIMITING DISTRIBUTIONS FOR CRITICAL MULTITYPE BRANCHING PROCESSES WITH DISCRETE TIME $\left({ }^{1}\right)$
}

\author{
BY \\ T. W. MULLIKIN
}

1. Introduction. We shall study a certain class of discrete branching processes with a finite or infinite number of types-a generalized branching process. We shall take as the mathematical representation of such a process a given analytic mapping of the unit sphere of a complex Banach space-a generalized probability-generating function. For a thorough probability-theoretic discussion of general branching processes we refer to the works of Harris $[10 ; 11]$ and Moyal [17].

A generalized branching process is a generalized, temporally homogeneous, Markov process $Z_{n}, n=0,1, \cdots$, where the range of the $Z_{n}$ is a set of nonnegative integer-valued set functions, and $Z_{0}$ is a given nonrandom function. An important classification of branching processes can be made in terms of the spectral radius $\sigma(M)$ of the expectation operator $M$ for one generation (assumed to be a bounded operator), according to whether $\sigma(M)<1, \sigma(M)=1$, or $\sigma(M)>1$. For a large class of processes, $\sigma(M)$ is in the point spectrum of $M$ with multiplicity 1 , and the expected value of $Z_{n}$ behaves like $[\sigma(M)]^{n}$ as $n \rightarrow \infty$. Except when $\sigma(M)=1$, limiting properties of $Z_{n}$ have been extensively studied $[3 ; 7 ; 10 ; 14 ; 24]$.

When $\sigma(M)=1$ for a process, it is said to be critical. It is known that except in certain special cases, such a process dies with probability 1 after a finite number of generations. Even so, the expected lifetime is infinite, and it is interesting to investigate population size at the $n$th generation, given that it is not zero.

Population size has been determined for critical discrete processes of one type by Yaglom [24] and for continuous time processes of a finite number of types by Sevast'yanov [23] and Čistyakov [2]. It has been shown that the population (if not empty) of the $n$th generation, or at time $t$, tends to contain $n$, or $t$, particles which are distributed among the various types in a definite ratio. Random fluctuations from this expected behavior are measured by certain conditional probability distributions defined for the $n$th generation. Each of these distributions has a limit of exponential type.

Received by the editors January 4, 1962.

(1) This research is sponsored by the United States Air Force under Project RAND Contract No. AF 49(638) 700 - monitored by the Directorate of Development Planning, Deputy Chief of Staff. Research and Technology. Hq. USAF. 
The purpose of this paper is to extend these results to a class of branching processes for discrete time with a finite or an infinite number of types. The $n$th generation is described by the $n$th iterate of the analytic mapping alluded to above. With enough assumptions about this mapping, the results we seek follow from an investigation of the iterates of this mapping near a fixed point.

In $\$ 2$ we define the branching processes we shall study and discuss the notation to be used. Properties of generating functions are extended in $\$ 3$ to generating functionals. In $\$ 4$ we study the iterates of the analytic mapping that defines a critical process. In $\S 5$ we give probabilistic meaning to the results of $\S 4$. Finally, in the last section we discuss two applications which illustrate why the class of processes studied is neither void nor uninteresting. One of these applications is concerned with processes having $k$ types of particles, and the other with the study of the neutron population in an idealized model of a spherical reactor, where the type of a neutron is the spherical shell in which it was produced by fission.

2. Critical, positively regular, branching processes. It is relatively easy to define and analyze a branching process when there are only a finite number of types of particles. In generalizing to processes with an infinite number of types, we select a Banach space and take a particular type of mapping of the unit sphere as the natural extension of the concept of a generating function. We avoid the delicate questions concerned with the derivation of this function from a given set of probabilities $[10 ; 11 ; 17]$.

To obtain results analogous to those for a finite number of types, we shall assume compactness and positivity of various mappings. For any specific branching process it may well be that the Banach space we choose here is not the natural one in which to prove compactness. At least we shall attain a certain degree of generality and give a method of proof which can perhaps be modified for other Banach spaces.

Let $X$ denote an abstract set-the set of types-and let $\Sigma$ denote a $\sigma$-field of subsets of $X$. We designate by $C(X, \Sigma)$ the complex Banach space which is the closure of the linear space of finite combinations of characteristic functions (indicator functions) of sets in $\Sigma$ with the norm

$$
\|s\|=\sup _{x \in X}|s(x)| \text {. }
$$

This is just the space of all bounded $\Sigma$-measurable functions.

The conjugate space to $C(X, \Sigma)$ is characterized by the bounded and finitely additive set functions defined on $\Sigma$ (p.258, Chapter III of [6]). That is, for each continuous linear functional $t^{*}$ on $C(X, \Sigma)$ there is a unique bounded, finitely additive, set function $\mu$ such that $t^{*}$ evaluated on the function $s$, written $t^{*}(s)$, is given by

$$
t^{*}(s)=\int_{X} s(x) d \mu(x)
$$


We refer to Chapter III of [6] for a development of the appropriate integration theory.

We denote by $R(X, \Sigma)$ the real Banach space consisting of real-valued functions in $C(X, \Sigma)$. This is a Banach lattice under the obvious partial ordering,

$$
s \geqq t \text { if and only if } s(x) \geqq t(x) \text { for all } x \text { in } X \text {. }
$$

We adopt the following notation:

$$
s>0, \quad \text { if } s \geqq 0 \quad \text { and } s \neq 0,
$$

and

$$
s \gg 0, \quad \text { if } s(x) \geqq \varepsilon>0 \quad \text { for all } x \text { in } X .
$$

The positive cone $P$ in $R(X, \Sigma)$ consists of those functions which are nonnegative. The dual cone $P^{*}$ in $R^{*}(X, \Sigma)$ is characterized by nonnegative and finitely additive set functions defined on $\Sigma$. We adopt in $P^{*}$ the following notation:

$$
\begin{gathered}
t^{*} \geqq 0 \text { if } t^{*}(p) \geqq 0 \text { for all } p \text { in } P, \\
t^{*}>0 \text { if } t^{*} \geqq 0 \text { and } t^{*}(p)>0 \text { for some } p \text { in } P,
\end{gathered}
$$

and

$$
t^{*} \gg 0 \text { if } t^{*}(p)>0 \text { for all } p \text { in } P, p \neq 0 \text {. }
$$

A bounded linear operator $T$ on $R(X, \Sigma)$ to $R(X, \Sigma)$ is said to be positive, and is written as

$$
T>0 \text {, if } T p \geqq 0 \text { for all } p \text { in } P \text {. }
$$

It is strictly positive, written

$$
T \gg 0 \text {, if } T p \gg 0 \text { for all } p \text { in } P, p \neq 0 \text {. }
$$

Some of the theory of positive operators to which we shall appeal depends upon the fact that $P$ has an interior, namely, those functions satisfying $p \gg 0$, and the fact that, by the Jordan Decomposition Theorem [6, p. 98], $P^{*}$ spans $R^{*}(X, \Sigma)$.

The open unit sphere in $C(X, \Sigma)$ is defined by

$$
S=\{s \mid s \text { is in } C(X, \Sigma) \text { and }\|s\|<1\} .
$$

For a function $s$, we define the function $|s|$ in the obvious way:

$$
|s|(x)=|s(x)|, \text { all } x \in X .
$$

The absolute value $|s|$ of a function should not be confused with the norm $\|s\|$. There is also the possibility of confusion of the unit scalar 1 with the constant function 1 in $C(X, \Sigma)$. To avoid this, we reserve the symbol $\mathbf{l}$ for the function

$$
\mathbf{l}(x)=1 \text {, for all } x \text { in } X \text {. }
$$


There is little possibility of confusing the zero function with the scalar 0 , so we use the same symbol for both.

We consider now a mapping $F$ of the unit sphere $S$ into $S$. We require that the following conditions be satisfied:

(a) $F$ maps $\Sigma$-measurable functions into $\Sigma$-measurable functions,

$$
\lim _{s \rightarrow 1} F(s)=1, \quad\|s\|<1,
$$

(c) $F$ is analytic in $S$, and all variations computed at $s=0$ satisfy

$$
\delta^{n} F\left(0 ; p_{1}, \cdots, p_{n}\right) \geqq 0, \text { all } p_{i} \in P, \quad n=0,1, \cdots .
$$

We refer to Hille and Phillips [13] for a treatment of analytic functions defined on a Banach space. The assumption of analyticity implies a power series expansion

$$
F(s)=\sum_{n=0}^{\infty} H_{n}(s), \quad H_{n}(s)=\frac{\delta^{n} F(0 ; s)}{n !}, \quad\|s\|<1 .
$$

Here $\delta^{n} F(0 ; s)$ is a bounded, homogeneous polynomial of degree $n$ in $s$, defined recursively by $H_{0}(s)=F(0)$ and

$$
\delta^{n+1} F(0 ; s)=\lim _{\lambda \rightarrow 0} \frac{\delta^{n} F(\lambda s ; s)-\delta^{n} F(0 ; s)}{\lambda}, \lambda \text { a complex scalar. }
$$

A continuous, symmetric, $n$-linear form is defined by

$$
H_{n}\left(h_{1}, \cdots, h_{n}\right) \equiv \frac{\delta^{n} F\left(0 ; h_{1}, \cdots, h_{n}\right)}{n !} .
$$

This means that $H_{n}$ is linear in each term, is unchanged by permutations of the $h_{i}$, and for some constant $K_{n}$,

$$
\left\|H_{n}\left(h_{1}, \cdots, h_{n}\right)\right\| \leqq K_{n} \prod_{i=1}^{n}\left\|h_{i}\right\| .
$$

If $h_{1}=\cdots=h_{k}=s$ and $h_{k+1}=\cdots=h_{n}=t$, we shall often abbreviate with the notation

$$
H_{n}\left(s^{k}, t^{n-k}\right) \equiv H_{n}(\overbrace{s, \cdots, s,}^{k}, \overbrace{t, \cdots, t}^{n-k}) .
$$

The series (2.15) can be differentiated term-wise to give

$$
\delta^{k} F(s ; h)=\sum_{n=k}^{\infty} \frac{n !}{(n-k) !} H_{n}\left(s^{n-k}, h^{k}\right) \text { for }\|s\|<1 .
$$

It should be remarked that $F(s)$ and $H_{n}(s)$ are functions in $C(X, \Sigma)$. We denote the value at the point $x$ in $X$ of $F(s)$, for example, by

$$
F(x, s) \equiv F(s)(x)
$$


We consider $F$ as defining a generalized branching process for particles with types specified by points in $X$. This is to be a temporally homogeneous Markov process $Z_{n}, n=0,1, \cdots$, where the range of the $Z_{n}$ is a set $L$ of functionals in $C^{*}(X, \Sigma)$ defined by additive, nonnegative and integer valued set functions on $\Sigma$. The initial state $Z_{0}$ is a nonrandom functional which is a finite sum of positive integer multiplies of functionals $e_{x}$ defined by

$$
e_{x}(s)=s(x) \text {. }
$$

The functional $F(x, s)$ defined on $S$ for fixed $x$ in $X$ is the generating functional for the first generation of progeny of a particle of type $x$.

To clarify the concept of a generating functional we take

$$
s=\sum_{i=1}^{k} \lambda_{i} \chi_{A_{i}}
$$

where $\lambda_{i}$ are complex numbers, $\left|\lambda_{i}\right|<1$, and $\chi_{A_{i}}$ are characteristic functions for disjoint sets $A_{i}$ such that $\bigcup_{i=1}^{k} A_{i}=X$. Then

$$
\begin{aligned}
F(x, s) & =\sum_{n=0}^{\infty} H_{n}(x, s) \\
& =\sum_{n=0}^{\infty} P_{n}\left(\lambda_{1}, \cdots, \lambda_{k}\right),
\end{aligned}
$$

where the $P_{n}$ are homogeneous polynomials of degree $n$ in $\lambda_{1}, \cdots, \lambda_{k}$, with coefficients which depend only on the $\chi_{A_{t}}$ and which are easily seen by use of (2.14) to be nonnegative. Since for $\lambda_{1}=\cdots=\lambda_{k}=1, s=1$ and $F(x, 1)=1$, $F\left(x, \sum_{i=1}^{k} \lambda_{i} \chi_{A_{i}}\right)$ is a generating function which, we assume, describes the progeny of a particle of type $x$ by distinguishing types of progeny only to the extent that they fall in the sets $A_{1}, \cdots, A_{k}$.

It is further assumed that if $Z_{n}=\sum_{i=1}^{k} r_{i} e_{x_{i}}$ for positive integers $r_{1}, \cdots, r_{k}$ and distinct points $x_{1}, \cdots, x_{k}$, then $Z_{n+1}$ is the sum of $r_{1}+\cdots+r_{k}$ independent random variables with generating functional

$$
\prod_{i=1}^{k}\left[F\left(x_{i}, s\right)\right]^{r i} \text {. }
$$

With these assumptions it is natural that the generating functional for the $n$th generation of progeny of a particle of type $x$ should be given by the $n$th iterate of the mapping $F$, i.e.,

$$
F_{n}(x, s)=F\left(x, F_{n-1}(s)\right),
$$

where $F_{n-1}(s)$ is of course a function.

With additional assumptions it will follow that $F_{n}$ satisfies the conditions imposed on $F$ so that it qualifies for defining a generating functional, but we shall not justify its defining the generating functional for $Z_{n}$. 
The mapping $F$ need not be analytic at $\mathbf{l}$; however we do require that $F$ satisfy the condition that

$$
\lim _{\lambda \uparrow 1} \delta^{k} F(\lambda \mathbf{l}, \mathbf{l}) \text { exist for } k=0,1,2 \text {, and } 3 \text {. }
$$

We show in the next section that this implies

$$
F(s)=1-M(1-s)+K(1-s)+O\left(\|\mathbf{1}-s\|^{3}\right), \quad\|s\|<1 .
$$

The operator $M$, the expectation operator, is bounded, linear and nonnegative. The operator $K$ is a nonnegative, continuous and homogeneous polynomial of degree 2 [13], i.e.,

$$
\begin{aligned}
K(\lambda h) & =\lambda^{2} K(h), \\
K(h+\lambda k) & =P_{0}(h, k)+\lambda P_{1}(h, k)+\lambda^{2} P_{2}(h, k) .
\end{aligned}
$$

where $\lambda$ is any complex number and $P_{0}, P_{1}$, and $P_{2}$ are bilinear operators independent of $\lambda$.

In infinite-dimensional Banach spaces the closed unit sphere $S$ is not compact in the norm topology, and this vitiates an immediate extension of proofs of results in finite dimensions. For some spaces a weaker topology makes the unit sphere compact, but none is known for general $C(X, \Sigma)$. For this reason it will be necessary to make certain assumptions about the mapping $M$.

We first of all assume continuity of $M$ on bounded monotone sequences. If $\left\{s_{n}\right\}$ is a sequence of functions in $R(X, \Sigma)$ and $s_{n+1} \geqq s_{n},\left\|s_{n}\right\| \leqq A$, then, since $\Sigma$ is a $\sigma$-field, $s_{n}$ will converge pointwise to a bounded measurable function $s$ in $R(X, \Sigma)[9]$. We assume that

$$
M(s)(x)=\lim _{n \rightarrow \infty} M\left(s_{n}(x)\right) \text { for all } x \text { in } X .
$$

This is not an unnatural assumption since we are considering $F(x, \cdot)$ to be a generating functional. If $F(x, s)$ is derived from a set of probabilities on the range $L$ of $Z_{1}$ in $C^{*}(X, \Sigma)$, then $M(s)(x)$ is an expectation, and (2.29) simply states the interchange of limit and expectation. It is perhaps possible to prove (2.29) from the assumptions (2.14), although we have been unable to do so.

The bounded linear transformation $M$ is positive in that it maps the positive cone $P$ into itself. It can be shown, using positivity of $M$ and the fact, noted earlier, that $P^{*}$ spans $R^{*}(X, \Sigma)$, that the spectral radius of $M$ is determined by the least upper bound $\sigma(M)$ of the spectrum of $M$ on the positive real axis [15]. Without further conditions on $M$ it is not possible generally to determine additional properties of $\sigma(M)$. Therefore, we make two additional assumptions on $M$ :

(a) For some integer $n_{0}, M^{n_{0}}$ is a compact operator.

(b) For some integer $n_{1}, M^{n_{1}}$ is a strictly positive operator. 
Here $M^{n}$ denotes the $n$th iterate of the operator $M$.

These properties of $M$ are sufficient for $\sigma(M)$ to be in the point spectrum of $M$ with unit multiplicity [16, Theorem 6.3]. In addition, for $\sigma(M)$ eigenfunctions $\phi$ of $M$ and $\phi^{*}$ of $M^{*}$ can be chosen to be strictly positive, i.e., $\phi$ is uniformly positive and $\phi^{*}(p)>0$ for all $p$ in $P, p>0$. The eigenvalue $\sigma(M)$ dominates the modulus of all other eigenvalues of $M$, and by the functional calculus [13],

$$
M^{n}=[\sigma(M)]^{n} P+Q_{n} .
$$

Here, if $\phi^{*}(\phi)=1, P$ is a projection given by

$$
P s=\phi^{*}(s) \phi, \quad P^{*} s^{*}=s^{*}(\phi) \phi^{*},
$$

and $Q_{n}$ is an operator satisfying

$$
\left\|Q_{n}\right\| \leqq A \alpha^{n} \text { for some fixed } A \geqq 0 \text { and } \alpha, 0 \leqq \alpha<1 .
$$

We shall say that the mapping $F$ defines a positively regular branching process -a PR process-provided that $F$ is not a linear mapping and that $F$ and $M$ satisfy the conditions (2.14), (2.26), (2.29) and (2.30).

Certain limiting properties of the process $Z_{n}$ as $n \rightarrow \infty$ are determined by the sequence of mappings $\left\{F_{n}\right\}$. As is well known, the behavior of this sequence depends primarily upon whether $\sigma(M)<1, \sigma(M)=1$, or $\sigma(M)>1$. Limiting distributions for various functions of $Z_{n}$ have been rather thoroughly investigated when $\sigma(M) \neq 1$. The main results of this paper are given in $\S 4$, where we shall consider only the critical case $\sigma(M)=1$ and extend to critical, positively regular, branching processes-a CPR process, a result known for discrete time processes of one type [24] and for continuous time processes of a finite number of types $[2 ; 23]$.

3. Properties of generating functionals. In the next section we shall need various properties of generating functionals that are obvious extensions of properties of ordinary probability generating functions. We derive these in the following sequence of lemmas and theorems, the validity of which seems obvious even though some proofs are lengthy.

We first establish some properties of symmetric $n$-linear forms of which $\delta^{n} F\left(0 ; h_{1}, \cdots, h_{n}\right)$ are examples.

LEMMA 1. Let $H_{n}$ be a symmetric n-linear form, continuous on the product space $\prod_{i=1}^{n} C(X, \Sigma)$ to $C(X, \Sigma)$. If $H_{n}$ is nonnegative on nonnegative functions, then it satisfies

and

$$
\left|H_{n}\left(h_{1}, \cdots, h_{n}\right)\right| \leqq H_{n}\left(\left|h_{1}\right|, \cdots,\left|h_{n}\right|\right) \leqq\left(\prod_{i=1}^{n}\left\|h_{i}\right\|\right) H_{n}(1, \cdots, 1)
$$

$$
H_{n}\left(h_{1}, \cdots, h_{n}\right)-H\left(g_{1}, \cdots, g_{n}\right)=\sum_{i=1}^{n} H_{n}\left(g_{1}, \cdots, g_{i-1}, h_{i}-g_{i}, h_{i+1}, \cdots, h_{n}\right)
$$


Proof. Suppose first that each function $h_{i}$ is a finite linear combination of $m_{i}$ characteristic functions. Since $H_{n}$ is linear in each argument, $H_{n}\left(h_{1}, \ldots, h_{n}\right)$ can be viewed as an $n$th degree polynomial in the $\prod_{i=1}^{n} m_{i}$ coefficients of the characteristic functions. Since the coefficients of the polynomial are nonnegative functions such as $H_{n}\left(\chi_{A_{1}}, \cdots, \chi_{A_{n}}\right),(3.1)$ follows immediately for such functions $h_{i}$. These functions are dense in $C(X, \Sigma)$, and $H_{n}$ and the norm are continuous functions so that (3.1) follows.

To prove (3.2), we observe by linearity that

$$
H_{n}\left(h_{1}, \cdots, h_{n}\right)=H\left(h_{1}-g_{1}, h_{2}, \cdots, h_{n}\right)+H\left(g_{1}, h_{2}, \cdots, h_{n}\right) .
$$

Repeating this $n$ times, we obtain (3.2) and the proof is complete.

Consequences of assumptions about the behavior of $F$ near $\mathbf{l}$ are given in the following theorem.

THEOREM 1. Let $F$ satisfy (2.14) and suppose that in $C(X, \Sigma)$ the functions $\delta^{k} F(\lambda \mathbf{l} ; \mathbf{l}), 0 \leqq \lambda<1, k=0,1,2,3$, have limits as $\lambda$ increases to 1 . Then the functions $\delta^{k} F(s ; h), k=0,1,2$, for $h \in C(X, \Sigma)$ have limits as $s \rightarrow 1,\|s\|<1$, and

$$
\lim _{s \rightarrow 1 ;\|s\|<1} \delta^{k} F(s ; h)=\sum_{n=k}^{\infty} \frac{n !}{(n-k) !} H_{n}\left(1^{n-k}, h^{k}\right) .
$$

If the limit of $\delta F(s ; h)$ is denoted by $M(h)$ and that of $\delta^{2} F(s ; h)$ by $2 K(h)$, then

$$
F(s)=1-M(1-s)+K(1-s)+O\left(\|1-s\|^{3}\right), \quad\|s\|<1,
$$

where $O\left(\|\mathbf{1}-s\|^{3}\right)$ is in $C(X, \Sigma)$ and satisfies $\left\|O\left(\|\mathbf{1}-s\|^{3}\right)\right\| \leqq A\|\mathbf{1}-s\|^{3}$ for a constant $A$ independent of $s,\|s\|<1$. ( The operator $M$ is nonnegative and linear with bound $\|M(1)\|$. The operator $K$ is a nonnegative, continuous, and homogeneous polynomial of degree 2.)

Proof. The function $\delta^{k} F(\lambda \mathbf{l} ; \mathbf{l})$, where $\lambda$ is a complex scalar satisfying $|\lambda|<1$, is given by $(2.20)$ as

$$
\delta^{k} F(\lambda \mathbf{l} ; \mathbf{l})=\sum_{n=k}^{\infty} \frac{n !}{(n-k) !} H_{n}(\mathbf{l}) \lambda^{n-k} .
$$

The coefficients in the power series are nonnegative functions, and an application of Abel's lemma shows that divergence of $\sum_{n=k}^{\infty}[n ! /(n-k) !] H_{n}(x, 1)$ for any $x \in X$ denies the existence of a limit for $\delta^{k} F(\lambda l ; 1)$ as $\lambda$ approaches 1 through real values. It follows readily that

$$
\lim _{\lambda \rightarrow 1} \delta^{k} F(\lambda \mathbf{l} ; \mathbf{l})=\sum_{n=k}^{\infty} \frac{n !}{(n-k) !} H_{n}(\mathbf{l}), \quad k=0,1,2,3 .
$$

We need also the fact that the sequence of partial sums

$$
\left\{s_{N}^{(k)}=\sum_{n=k}^{N}[n ! /(n-k) !] H_{n}(\mathbf{l})\right\}
$$


converges uniformly as $N \rightarrow \infty$, since (3.7) guarantees only that it converges pointwise to a function $s^{(k)}$ in $C(X, \Sigma)$. We prove this for $k=0,1$, and 2 by observing that with the series representations of $s^{(k)}$ and $\delta^{k} F(\lambda l ; 1)$, we have

$$
\begin{aligned}
& \left\|s^{(k)}-s_{N}^{(k)}\right\| \leqq\left\|s^{(k)}-\delta^{k} F(\lambda \mathrm{l} ; \mathrm{l})\right\|+\left\|\sum_{n=k}^{N} \frac{n !}{(n-k) !} H_{n}(\mathrm{l})\left(1-\lambda^{n-k}\right)\right\| \\
& +\left\|\sum_{n=N+1}^{\infty} \frac{n !}{(n-k) !} H_{n}(\mathrm{l}) \lambda^{n-k}\right\| \\
& \leqq\left\|s^{(k)} \delta^{k} F(\lambda 1 ; \mathbf{1})\right\|+(1-\lambda)\left\|\sum_{n=k+1}^{\infty} \frac{n !}{(n-k-1) !} H_{n}(\mathbf{1})\right\| \\
& +\left\|\sum_{n=N+1}^{\infty} \frac{n !}{(n-k) !} H_{n}(\mathrm{l}) \lambda^{n-k}\right\| \text {. }
\end{aligned}
$$

The coefficient of $(1-\lambda)$ in this last expression is bounded because of (3.7) and the restriction of $k$ to 0,1 , or 2 . Now for any given $\varepsilon>0$, we can choose $\lambda_{0}(\varepsilon)$ so that the first term on the right of (3.8) is less that $\varepsilon / 3$ for $1 \geqq \lambda>\lambda_{0}(\varepsilon)$ because of (3.7). The second term can be made less than $\varepsilon / 3$ by choosing $\lambda$ sufficiently close to 1 . Having chosen a $\lambda_{0}<1$ satisfying these two conditions, we can make the last term less than $\varepsilon / 3$ for all $N>N_{0}\left(\varepsilon, \lambda_{0}\right)$ by virtue of the convergence of the power series. Therefore $s_{N}^{(k)} \rightarrow s^{(k)}$ uniformly.

For arbitrary $h$ in $C(X, \Sigma)$ and $k=0,1$, or 2, we have proved, by Lemma 1 and the uniform convergence, that

$$
\left\|\sum_{n=N}^{M} \frac{n !}{(n-k) !} H_{n}\left(1^{n-k}, h^{k}\right)\right\| \leqq\|h\|^{k}\left\|_{n=N}^{M} \frac{n !}{(n-k) !} H_{n}(1)\right\| \rightarrow 0 \text { as } N, M \rightarrow \infty .
$$

Therefore, the partial sums $\sum_{n=k}^{N} n ! /(n-k) ! H_{n}\left(1^{n-k}, h^{k}\right)$ form a Cauchy sequence in $C(X, \Sigma)$ and the series converges.

Finally, by the series representation (2.15) of $\delta^{k} F$ for $\|s\|<1$ and by the inequalities in Lemma 1, we have

$$
\begin{aligned}
& \left\|\delta^{k} F(s ; h)-\sum_{n=k}^{\infty} \frac{n !}{(n-k) !} H_{n}\left(1^{n-k}, h^{k}\right)\right\| \\
& \quad \leqq\|1-s\|\|h\|^{k}\left\|_{n=k+1}^{\infty} \frac{n !}{(n-k-1) !} H_{n}(1)\right\| \text { for } k=0,1,2 .
\end{aligned}
$$

This proves (3.4).

To prove (3.5) we observe that, by analyticity of $F$, the function defined on $0 \leqq \lambda<1$ by $F(s+\lambda(1-s)),\|s\|<1$, is twice continuously differentiable, and by definition of the variations of $F$,

$$
\frac{d^{k}}{d \lambda^{k}} F\left(s+\lambda(1-s) \equiv \delta^{k} F(s ; 1-s), \quad k=1,2 .\right.
$$


With the standard notion of the Riemann integral of vector valued functions [13], the result (3.4) proved above, and the definition of the operators $M$ and $K$,

$$
\begin{aligned}
M(1-s)-\delta F(s & +\mu(1-s) ; 1-s) \\
& =\int_{\mu}^{1} \delta^{2} F(s+\lambda(1-s) ; 1-s) d \lambda \\
& =(1-\mu) 2 K(1-s)+O\left(\|1-s\|^{3}\right),
\end{aligned}
$$

where $O\left(\|1-s\|^{3}\right)$ denotes a function in $C(X, \Sigma)$ such that

$$
\frac{\left\|O\left(\|1-s\|^{3}\right)\right\|}{\|1-s\|^{3}} \leqq A<+\infty, A \text { independent of } s,\|s\|<1 \text {. }
$$

By (3.10) with $k=2$ and $h=1-s$, the last statement in (3.12) is possible; a possible constant $A$ in (3.13) is simply $\left\|\sum_{n=3}^{\infty} n ! /(n-3) ! H_{n}(1)\right\|$. If we integrate (3.12) from $\mu=0$ to $\mu=1$, we obtain (3.5) and thus complete the proof.

It is an immediate consequence of Lemma 1 that $F$ satisfying (2.14) is a monotone mapping on positive functions. That is,

$$
0 \leqq s \leqq t \text { implies } 0 \leqq F(s) \leqq F(t) .
$$

Many of the results that follow depend upon other inequalities which are extensions of properties of generating functions.

THEOREM 2. Let $F$ satisfy the conditions of Theorem 1. Then for $\|s\| \leqq 1$

$$
|F(s)| \leqq F(|s|)
$$

and

$$
1-F(|s|) \geqq M(1-|s|)-K(1-|s|) .
$$

For $\|s\|<1$ and $\|t\| \leqq 1$,

$$
|F(t)-F(s)| \leqq M(|t-s|),
$$

and if $|s| \ll|t|$, equality is possible in (3.17) only if $F$ is linear.

Proof. Directly from Lemma 1 and the series representation (2.15) of $F$, we get (3.15).

For real-valued $s, 0 \leqq s \leqq 1$, the function $\delta^{2} F(s+\lambda(1-s) ; 1-s), 0 \leqq \lambda \leqq 1$, is nondecreasing with increasing $\lambda$ because

$$
\begin{aligned}
\delta^{2} F(s+\lambda(1-s) ; & 1-s) \\
= & \sum_{n=2}^{\infty} n(n-1) H_{n}\left([s+\lambda(1-s)]^{n-2},[1-s]^{2}\right),
\end{aligned}
$$

and $H_{n}\left([s+\lambda(1-s)]^{n-2},[1-s]^{2}\right)$ is a polynomial in $\lambda$ of degree $n-2$ with nonnegative coefficients. Therefore from (3.12) we obtain 


$$
M(\mathbf{I}-|s|)-\delta F(|s|+\mu(\mathbf{I}-|s|) ; \mathbf{I}-|s|) \leqq(1-\mu) 2 K(\mathbf{I}-|s|),
$$

and an additional integration on $\mu$ from 0 to 1 gives (3.16).

The results of Lemma 1 give

$$
\begin{aligned}
|F(t)-F(s)| & \leqq \sum_{n=0}^{\infty}\left|H_{n}(t)-H_{n}(s)\right| \\
& \leqq \sum_{n=1}^{\infty} \sum_{i=1}^{n} H_{n}\left(|t|^{n-i},|s|^{i-1},|t-s|\right) \\
& \leqq \sum_{n=1}^{\infty} \sum_{i=1}^{n}\|s\|^{i-1}\|t\|^{n-i} H_{n}\left(1^{n-1},|t-s|\right) \\
& \leqq \sum_{n=1}^{\infty} n H_{n}\left(1^{n-1},|t-s|\right)=M(|t-s|) .
\end{aligned}
$$

If $|s|<|t|$, then equality in (3.20) implies

$$
0=H_{n}\left(1^{n-1},|t-s|\right) \geqq H_{n}\left(1^{n-1},|t|-|s|\right) \geqq 0, \text { for } n \geqq 2,
$$

since factors appear in (3.20) which are powers of $\|s\|<1$. But since $|t|-|s| \gg 0$ this implies that $H_{n}\left(1^{n-1}, h\right)=0, n \geqq 2$, for all $h$ in $C(X, \Sigma)$, and hence that $F$ is linear. This completes the proof.

In the investigation of iterates of generating functionals which define positively regular processes we shall need the following result.

Theorem 3. Let $F$ define a PR process. Then

$$
\|F(s)\|<1 \text { for }\|s\|<1 \text {. }
$$

Iterates $F_{n}$ of $F$ are then analytic on $S$, continuous on $S$, and satisfy conditions imposed on $F$. For $n \geqq n_{1}$ of (2.30) we have

$$
\left\|F_{i n}(s)\right\|<1 \text { for }\|s\| \leqq 1, \quad s \neq 1 .
$$

Proof. By Theorem 2, the monotonicity of $F$, and the quadratic nature of $K$, we have for $0 \leqq \lambda \leqq 1$

$$
\begin{aligned}
1-|F(s)| & \geqq 1-F(|s|) \geqq 1-F(|s|+\lambda(1-|s|)) \\
& \geqq(1-\lambda)[M(1-|s|)-(1-\lambda) K(1-|s|)] .
\end{aligned}
$$

Now if $1-|s| \gg 0$ there is an $\alpha>0$ such that $1-|s| \geqq \alpha \phi \gg 0, M \phi=\sigma(M) \phi$, by assumption (2.30). Since $K(1-|s|)$ is bounded, there clearly exists a $\lambda<1$ such that

$$
1-|F(s)| \geqq(1-\lambda)[\alpha \sigma(M) \phi-(1-\lambda) K(1-|s|)] \gg 0 .
$$

This proves (3.22). 
The mapping $F$ takes the open sphere $S$ into itself and hence into the domain of analyticity of $F$. It follows readily that

$$
\begin{aligned}
F_{1}(s) & =F(s), \\
F_{n+1}(s) & =F\left(F_{n}(s)\right)
\end{aligned}
$$

defines a sequence of analytic mappings of $S$ satisfying the assumptions made on $F$. In particular, we have the expectation operator $M_{n}$ given by

$$
M_{n}(h)=M^{n}(h) \text {. }
$$

The mappings $F_{n}$ are analytic on $S$, and by Theorem 1 and (3.15) of Theorem 2, continuous on the closed sphere $S$. If, for $F_{n}$, we denote the limit of $\delta^{2} F_{n}(s ; h)$ at $s=1$ by $K_{n}(h)$, then as in (3.24) we have

$$
1-\left|F_{n}(s)\right| \geqq(1-\lambda)\left[M^{n}(1-|s|)-(1-\lambda) K_{n}(1-|s|)\right] .
$$

By (2.30), $M^{n}(1-|s|) \gg 0$ if $n \geqq n_{1}$, and hence there exists a $\lambda<1$ such that

$$
1-\left|F_{n}(s)\right| \gg 0 \text { for }\|s\| \leqq 1, \quad s \neq \mathbf{l} .
$$

This completes the proof.

4. Iterates of generating functionals. We shall now prove several results about iterates of the mapping $F$ of $S$ into $S$, where $F$ satisfies the conditions required to generate a CPR process as defined in $\$ 2$. One of these results is known and others are motivated by the treatment of branching processes of one type described in Harris [11], where techniques of Fatou [8] are used.

Throughout this section we reserve the symbols $\phi$ and $\phi^{*}$ to denote strictly positive eigenfunctions of the operators $M$ and $M^{*}$ respectively. Normalization of $\phi$ and $\phi^{*}$ will be specified subject to the condition that $\phi^{*}(\phi)=1$.

For completeness we now prove a wellknown result for branching processes $[7 ; 11 ; 21 ; 22]$.

\section{THEOREM 4. For a CPR process,}

$$
\lim _{n \rightarrow \infty} F_{n}(s)=1 \text {, uniformly in } s,\|s\| \leqq 1 .
$$

Proof. We first show that there is no solution to

$$
s=F(s), \quad\|s\|<1 .
$$

For if such a solution exists, then by Theorem 2 and nonlinearity of $F$,

$$
|\mathbf{l}-s|=|\mathbf{l}-F(s)|<M(|\mathbf{1}-s|) \text {. }
$$

From (4.3) and the positivity of $\phi^{*}$, we have

$$
\phi^{*}[M(|1-s|)-|1-s|]>0 .
$$


But in fact equality must hold in (4.4) since $M^{*} \phi^{*}=\phi^{*}$, and we are led to a contradiction.

In particular, we note that

$$
F(0)>0,
$$

and since $F$ is monotone, the sequence $\left\{F_{n}(0)\right\}$ is monotone in the positive cone in $S$. The functions $F_{n}(0)$ are bounded by 1 , and so they will converge pointwise on $X$ to a unique nonnegative function $p, p \neq 0$, in $C(X, \Sigma)$. The monotonicity of $F$ and the inequality $p \geqq F_{n}(0)$ gives $F(p) \geqq p$. By this and (3.17) of Theorem 2, iterated $n_{0}$ times, we have

$$
p-F_{n+n_{0}}(0) \leqq M^{n_{0}}\left(p-F_{n}(0)\right)
$$

If $n_{0}$ is defined by (2.30), $M^{n_{0}}$ is a compact operator, and a subsequence of $\left\{M^{n_{0}}\left(p-F_{n}(0)\right\}\right.$ converges uniformly to a limit $q$. We have assumed in (2.29) that $M$, and hence $M^{n_{0}}$, is continuous on monotone sequences. Since $p-F_{n}(0)$ converges pointwise to 0 , so does $M^{n_{0}}\left(p-F_{n}(0)\right)$, and therefore the limit function $q$ must be 0 for any uniformly convergent subsequence.

This shows that $F_{n}(0)$ converges uniformly to $p$, and since $F_{n+1}(0)=F\left(F_{n}(0)\right)$, the continuity of $F$ gives

$$
F(p)=p, \quad 0<p \leqq 1 .
$$

We have already shown that $p$ cannot satisfy (4.7) and $p \ll 1$. It follows readily from

$$
1-p \leqq M(1-p)
$$

that equality must hold and that $1-p=\alpha \phi, \alpha \geqq 0$. But $\phi$ is uniformly positive and $p(x)=1$ for some $x$ in $X$ so that $\alpha=0$ and $p=1$.

By Lemma 1, we have the inequality

$$
|F(s)-F(0)| \leqq \sum_{n=1}^{\infty} H_{n}(|s|) \leqq F(|s|)-F(0) \leqq 1-F(0)
$$

A similar result holds for each $F_{n}$, so that

$$
\left|1-F_{n}(s)\right| \leqq\left|1-F_{n}(0)\right|+\left|F_{n}(s)-F_{n}(0)\right| \leqq 2\left|1-F_{n}(0)\right| .
$$

This proves uniformity of convergence in $s$ and completes the proof.

We now show that the sequence $F_{n}(0)$ tends to $\mathbf{l}$ along a given direction.

THEOREM 5. For a CPR process

$$
\phi^{*}\left(\mathbf{l}-F_{n}(0)\right)>0 \text { for } n \geqq 0,
$$

and

$$
\lim _{n \rightarrow \infty} \frac{1-F_{n}(0)}{\phi^{*}\left(1-F_{n}(0)\right)}=\phi, \quad \phi^{*}(\phi)=1
$$


Proof. For brevity we define $\Delta_{n}$ by

$$
\Delta_{n} \equiv 1-F_{n}(0) \text {. }
$$

By monotonicity of $F$ and (3.22) of Theorem 3, we have

$$
0 \ll \Delta_{n+1} \leqq \Delta_{n}
$$

and hence (4.11).

With $s=F_{n}(0)$ in (3.16) we have, by (4.14) and the homogeneous quadratic nature of $K$,

$$
\frac{\Delta_{n}}{\left\|\Delta_{n}\right\|} \geqq \frac{\Delta_{n+1}}{\left\|\Delta_{n}\right\|} \geqq M\left(\frac{\Delta_{n}}{\left\|\Delta_{n}\right\|}\right)-\left\|\Delta_{n}\right\| K\left(\frac{\Delta_{n}}{\left\|\Delta_{n}\right\|}\right) .
$$

By assumption (2.30) $M^{n_{0}}$ is a compact operator for some integer $n_{0} \geqq 1$. Since $M^{n_{0}}$ is also a nonnegative operator, we can apply it to (4.15) to obtain a sequence of nonnegative functions $\left\{p_{n}\right\}$,

$$
p_{n} \equiv M^{n_{0}}\left(\frac{\Delta_{n}}{\left\|\Delta_{n}\right\|}\right)
$$

which satisfy (by (4.15))

$$
M p_{n} \leqq p_{n}+\left\|\Delta_{n}\right\| M^{n_{0}}\left[K\left(\frac{\Delta_{n}}{\left\|\Delta_{n}\right\|}\right)\right]
$$

and which lie in a compact subset of $S$.

Every subsequence of $\left\{p_{n}\right\}$ has a convergent subsequence with a limit $p$ which satisfies

$$
M p \leqq p, \quad\|p\|=1,
$$

since in (4.17) $\left\|\Delta_{n}\right\| \rightarrow 0$, by Theorem 4 , and

$$
\left\|M^{n_{0}}\left[K\left(\frac{\Delta_{n}}{\left\|\Delta_{n}\right\|}\right)\right]\right\|
$$

is $\|M\|^{n}\|K(\mathrm{l})\|$. As shown several times before, equality must hold in (4.18) and therefore

Now by (3.5)

$$
\lim _{n \rightarrow \infty} M^{n_{0}}\left(\frac{\Delta_{n}}{\left\|\Delta_{n}\right\|}\right)=\phi, \quad\|\phi\|=1, \quad M \phi=\phi .
$$

$$
\Delta_{n+1}=M\left(\Delta_{n}\right)-K\left(\Delta_{n}\right)+O\left(\left\|\Delta_{n}\right\|^{3}\right)
$$

or by iteration

$$
\Delta_{n+n_{0}}=M^{n_{0}}\left(\Delta_{n}\right)-\sum_{i=0}^{n_{0}-1} M^{n_{0}-i-1}\left[K\left(\Delta_{n+i}\right)+O\left(\left\|\Delta_{n+i}\right\|^{3}\right)\right] .
$$

Dividing by $\left\|\Delta_{n}\right\|$, we get from (4.14), (4.19), and the quadratic nature of $K$,

$$
\lim _{n \rightarrow \infty} \frac{\Delta_{n+n 0}}{\left\|\Delta_{n}\right\|}=\phi, \quad\|\phi\|=1 .
$$


Therefore

$$
\lim _{n \rightarrow \infty} \frac{\left\|\Delta_{n+n_{0}}\right\|}{\left\|\Delta_{n}\right\|}=1
$$

and finally

$$
\lim _{n \rightarrow \infty} \frac{\Delta_{n}}{\left\|\Delta_{n}\right\|}=\phi, \quad\|\phi\|=1, \quad M \phi=\phi,
$$

as well as

$$
\lim _{n \rightarrow \infty} \frac{\Delta_{n}}{\phi^{*}\left(\Delta_{n}\right)}=\phi, \quad \phi^{*}(\phi)=1 .
$$

This completes the proof.

We are now in a position to prove a more refined result. By applying the linear functional $\phi^{*}$, we reduce the function sequence $\left\{F_{n}(s)\right\}$ to a scalar sequence to which the techniques of Fatou can be applied [11].

THEOREM 6. For a CPR process,

$$
\lim _{n \rightarrow \infty} n\left(\mathbf{I}-F_{n}(s)\right)=\frac{1}{\phi^{*}[K(\phi)]} \phi
$$

for $\|s\|<1, \phi^{*}(\phi)=1$.

Proof. Recalling (4.13), we set $s=F_{n}(0)$ in (3.5) and apply the linear functional $\phi^{*}$ to obtain

$$
\phi^{*}\left(\Delta_{n+1}\right)=\phi^{*}\left(\Delta_{n}\right)-\phi^{*}\left(K\left(\Delta_{n}\right)+O\left(\left\|\Delta_{n}\right\|^{3}\right)\right) .
$$

Since $K$ is quadratic this gives

$$
\lim _{n \rightarrow \infty} \frac{\phi^{*}\left(\Delta_{n+1}\right)}{\phi^{*}\left(\Delta_{n}\right)}=1 .
$$

Also by Theorem 5, the quadratic nature of $K$, and (4.27), we have

$$
\lim _{n \rightarrow \infty} \frac{1}{\phi^{*}\left(\Delta_{n}\right)}\left[1-\frac{\phi^{*}\left(\Delta_{n+1}\right)}{\phi^{*}\left(\Delta_{n}\right)}\right]=\phi^{*}[K(\phi)] .
$$

We consider the identity

$$
\frac{1}{\phi^{*}\left(\Delta_{n+1}\right)} \equiv \frac{1}{\phi^{*}\left(\Delta_{n}\right)}+\frac{1}{\phi^{*}\left(\Delta_{n}\right)}\left[1-\frac{\phi^{*}\left(\Delta_{n+1}\right)}{\phi^{*}\left(\Delta_{n}\right)}\right] \frac{\phi^{*}\left(\Delta_{n}\right)}{\phi^{*}\left(\Delta_{n+1}\right)}
$$

For brevity, we denote the last term in (4.30) by $A_{n}$ and observe from (4.28) and (4.29) that

$$
\lim _{n \rightarrow \infty} A_{n}=\phi^{*}[K(\phi)] .
$$

Adding (4.30) for values of $n$ from 1 to $N-1$ and dividing by $N$, we obtain

$$
\frac{1}{N \phi^{*}\left(\Delta_{N}\right)}=\frac{1}{N \phi^{*}\left(\Delta_{1}\right)}+\frac{1}{N} \sum_{n=1}^{N-1} A_{n} \text {. }
$$


In view of (4.31), we have

$$
\lim _{N \rightarrow \infty} \frac{1}{N \phi^{*}\left(\Delta_{N}\right)}=\phi^{*}[K(\phi)]
$$

Combining this result with (4.12) we find that

$$
\lim _{n \rightarrow \infty} n \Delta_{n}=\lim _{n \rightarrow \infty} \frac{\Delta_{n}}{\phi^{*}\left(\Delta_{n}\right)} \lim _{n \rightarrow \infty} n \phi^{*}\left(\Delta_{n}\right)=\frac{1}{\phi^{*}\left[\frac{K(\phi)}{K}\right]} \phi,
$$

which proves our result for $s=0$.

Next consider real-valued functions $s, 0<s<1$. Since $\lim _{n \rightarrow \infty} F_{n}(0)=1$, there is an integer $m$ for which

$$
F_{m}(0) \geqq s
$$

Then

$$
1-F_{n+m}(0) \leqq 1-F_{n}(s) \leqq 1-F_{n}(0)
$$

proves (4.26) for positive real-valued $s$.

Finally, for any $s,\|s\|<1$.

$$
\begin{aligned}
& \left|\frac{\phi}{\phi^{*}[K(\phi)]}-n\left(1-F_{n}(s)\right)\right| \leqq\left|\frac{\phi}{\phi^{*}[K(\phi)]}-n \Delta_{n}\right|+n\left|F_{n}(0)-F_{n}(s)\right| \\
& \leqq\left|\frac{\phi}{\phi^{*}[K(\phi)]}-n \Delta_{n}\right|+n\left|F_{n}(|s|)-F_{n}(0)\right| \\
& \rightarrow 0 \text { as } n \rightarrow \infty \text {, }
\end{aligned}
$$

where we have used the inequality $\left|F_{n}(s)-F_{n}(0)\right| \leqq F_{n}(|s|)-F_{n}(0)$ discussed in (4.9) and (4.10). This completes the proof.

We have now shown that the sequence of analytic mappings $n\left(1-F_{n}\right)$ tends to a constant in the limit. In the next section we shall give probabilistic interpretations to this result. We shall also determine certain limiting distributions by Laplace transform techniques.

In preparation for this, we let $p$ be a nonnegative function in $C(X, \Sigma)$ and we let $A_{i}, i=1, \cdots, k$, be disjoint sets in $\Sigma$ such that $\bigcup_{i=1}^{k} A_{i}=X$. We denote by $p_{i}$ the function $p \chi_{A_{i}}$, i.e.,

$$
p_{i}(x)= \begin{cases}p(x) & \text { for } x \text { in } A_{i} \\ 0 & \text { otherwise. }\end{cases}
$$

A sequence $\left\{s_{i q}\right\}$ is defined in $S$ for complex scalars $\lambda_{i}$ by

$$
s_{n}=\exp \left[-\frac{1}{n} \sum_{i=1}^{k} \lambda_{i} p_{i}\right], \quad n \geqq 1, \quad \operatorname{Re}\left(\lambda_{i}\right)>0 .
$$

To avoid cumbersome notation in the following theorem, we shall often denote the vector $\left(\lambda_{1}, \cdots, \lambda_{k}\right)$ by $\lambda$ and the function $\sum_{i=1}^{k} \lambda_{i} p_{i}$ by $\lambda p$. 
THEOREM 7. For a CPR process, the function $G_{n}$ defined by

$$
G_{n}(p, \lambda)=n\left(1-F_{n}\left(s_{n}\right)\right)
$$

is for $n \geqq n_{1}$ of (2.30), an analytic function of the $k$ complex variables, $\lambda_{1}, \cdots, \lambda_{k}$, in the domain $\operatorname{Re}\left(\lambda_{i}\right)>0$. Also

$$
\lim _{n \rightarrow \infty} G_{n}(p, \lambda)=\frac{\sum_{i=1}^{k} \lambda_{i} \phi^{*}\left(p_{i}\right)}{1+\phi^{*}[K(\phi)] \sum_{i=1}^{k} \lambda_{i} \phi^{*}\left(p_{i}\right)} \phi ; \quad \phi^{*}(\phi)=1
$$

uniformly in any compact subset of $\operatorname{Re}\left(\lambda_{i}\right)>0$.

Proof. Since (4.41) is trivial for $p=0$, we consider $p>0$. We select a bounded $\lambda$-domain $\Lambda$ by requiring

$$
0<\varepsilon<\operatorname{Re}\left(\lambda_{i}\right) \text { and }\left|\lambda_{i}\right|<R, \quad \varepsilon<R<\infty, \quad i=1, \cdots, k .
$$

Since $p$ is a fixed nonnegative function, there is a constant $c$ independent of $\lambda$ in $\Lambda$ and $n$ such that

$$
n\left\|1-s_{i 1}\right\| \leqq C<\infty, \quad n \geqq 0 .
$$

For brevity, we define the function $\Delta_{j, n}$ by

$$
\begin{aligned}
\Delta_{0, n} & =n\left(1-s_{n}\right), \\
\Delta_{j, n} & =n\left(1-F_{j}\left(s_{n}\right)\right), \quad 0<j \leqq n .
\end{aligned}
$$

By Theorem 3, $F_{n_{1}}\left(s_{n}\right)$ is interior to $S$ and hence in the domain of analyticity of all $F_{j}, j \geqq 1$. To conclude that $G_{n}$, or $\Delta_{n, n}$, is an analytic function on $\Lambda$ to $C(X, \Sigma)$ for $n \geqq n_{1}$, it suffices to show that $F_{n_{1}}\left(e^{-\lambda p}\right), \lambda p=\sum_{i=1}^{k} \lambda_{i} p_{i}$, is analytic in $\operatorname{Re}\left(\lambda_{i}\right)$ $>0$ for any $p>0$. If we let $H_{j}$ stand for $\delta^{j} F_{n_{1}}(0)$ and take $s=\exp \left(-\sum_{i=1}^{k} \lambda_{i} p_{i}\right)$, th :n by Theorem 3 and Lemma 1 ,

$$
\sum_{j=1}^{\infty} j\left|H_{j}\left(s^{j-1},-p_{i} s\right)\right| \leqq \sum_{j=1}^{\infty} j H_{j}\left(1, p_{i}\right)=M^{n^{1}}\left(p_{i}\right) .
$$

This series on the left converges absolutely and uniformly. It follows easily from Lemma 1 and Theorem 1 (restated with $F$ replaced by $F_{n_{1}}$ ) that, with $t=e^{-\Delta p_{i}} s, \operatorname{Re}\left(\lambda_{i}+\Delta\right)>0$,

$$
\begin{aligned}
\mid \frac{F_{n_{1}}(t)-F_{n_{1}}(s)}{\Delta}-\sum_{j=1}^{\infty} j H_{j}\left(s^{j-1},-p_{i} s\right) \\
\left.\leqq|\Delta| M^{n_{1}}\left(p_{i}\right)+6 K_{n_{1}}\left(p_{i}\right)\right] \rightarrow 0 \text { as }|\Delta| \rightarrow 0,
\end{aligned}
$$

where $K_{n_{1}}\left(p_{i}\right)$ is the limit at $s=1$ of $\delta^{2} F_{n_{1}}\left(s ; p_{i}\right)$. Therefore the partial derivatives of $F_{n_{1}}$ exist for $\operatorname{Re}\left(\lambda_{i}\right)>0$ and $p_{i} \geqq 0$, and $F$ is analytic in the complex variables 
$\lambda_{1}, \cdots, \lambda_{k}\left[13\right.$, p. 107]. Actually, the same argument applies for any $F_{j}\left(s_{n}\right)$ and it is not necessary to appeal to (2.23) of Theorem 3.

By Theorem 2 and properties (2.31), (2.32) and $\sigma(M)=1$ of $M$, we have

$$
\begin{aligned}
\left\|\Delta_{j, n}\right\| & \leqq\left\|n M^{j}\left(\left|\mathbf{l}-s_{n}\right|\right)\right\|=\left\|n \phi^{*}\left(1-s_{n}\right) \phi+n Q_{j}\left(1-s_{n}\right)\right\| \\
& \leqq n\left\|\mathbf{l}-s_{n}\right\|\left(\left\|\phi^{*}\right\|+A \alpha^{j}\right),
\end{aligned}
$$

where $A$ and $\alpha$ are independent of $n$ and $\lambda, 0 \leqq x<1$. Therefore on $\Lambda$ we have a double sequence $\left\{\Delta_{j, n}\right\}$ of analytic functions, and by (4.42),

$$
\left\|\Delta_{j, n}\right\| \leqq B
$$

where $B$ is a constant independent of $\lambda$ in $\Lambda$, and $0 \leqq j \leqq n$.

By a generalized theorem of Vitali $([13, \S 3.15]$ and $[1$, p. 41$])$, the convergence of $\left\{\Delta_{n, n}\right\}$ on $\Lambda$ will be proved if convergence is demonstrated on a real neighborhood in $\Lambda$. Therefore we enlarge $\Lambda, \mathrm{i}$ necessary, so that $\lambda$ restricted by

$$
\phi^{*}[K(\phi)] \sum_{i=1}^{k} \lambda \phi^{*}\left(p_{i}\right)<1, \quad 0<\varepsilon<\lambda_{i}<R,
$$

defines a relatively open real set in $\Lambda$. We shall prove (4.41) on this set $N$.

We first of all show that $\phi^{*}\left(\Delta_{m, n}\right)$ is positive on the set (4.49) for $n$ sufficiently large. By Theorem 2, with $s=F_{j}\left(s_{n}\right)$, and by the quadratic nature of $K$,

$$
\Delta_{j+1, n} \geqq M \Delta_{j, n}-\frac{1}{n} K\left[M^{j}\left(\Delta_{0, n}\right)\right] .
$$

Applying the positive functional $\phi^{*}$, summing on $j$ from 0 to $m-1$, and using $n\left(1-s_{n}\right)<\lambda p$ (recalling that $\left.\lambda p=\sum_{i=1}^{k} \lambda_{i} p_{i}\right)$, we obtain

$$
\phi^{*}\left(\Delta_{m, n}\right) \geqq \phi^{*}\left(\Delta_{0, n}\right) \frac{1}{n}-\sum_{j=0}^{n} \phi^{*}\left(K\left[M^{j}(\lambda p)\right],\right.
$$

where the upper limit on the last sum has been increased from $m-1$ to $n$. Now by (2.31) for $\sigma(M)=1$ and by the quadratic nature of $K$, the sequence

$$
\left\{\phi^{*}\left(K\left[M^{j}(\lambda p)\right]\right)\right\}
$$

has the limit $\left[\phi^{*}(\lambda p)\right]^{2} \phi^{*}[K(\phi)]$, and so does the average appearing in (4.51). Therefore, since $\Delta_{0, n} \rightarrow \lambda p$ as $n \rightarrow \infty$, we have (by (4.49) and $\phi^{*}(\lambda p)>0$ )

$$
\lim _{n \rightarrow \infty} \inf \phi^{*}\left(\Delta_{m, n}\right) \geqq \phi^{*}(\lambda p)\left(1-\phi^{*}[K(\phi)]\right)>0 .
$$

Since the right-hand side of (4.51) is independent of $m, \phi^{*}\left(\Delta_{m, n}\right)$ can be bounded away from 0 for $n$ sufficiently large, independent of $m$.

By repeated application of (3.5) we obtain

$$
\Delta_{j+1, n}=M^{j}\left(\Delta_{0, n}\right)-\frac{1}{n} \sum_{k=0}^{j} M^{j-k}\left[K\left(\Delta_{k, n}\right)-\frac{1}{n} O\left(\left\|\Delta_{k, n}\right\|^{3}\right)\right]
$$


We apply (2.31) and (2.32) with $\sigma(M)=1$ and $Q_{0}=M-P$, and identify the terms containing $\phi$ as simply $\phi^{*}\left(\Delta_{j+1, n}\right)$ to get, from (4.53),

$$
\begin{aligned}
\Delta_{j+1, n}= & \phi^{*}\left(\Delta_{j+1, n}\right) \phi+Q_{j}\left(\Delta_{0, n}\right) \\
& -\frac{1}{n} \sum_{k=0}^{j} Q_{j-k} K\left[\left(\Delta_{k, n}\right)-\frac{1}{n} O\left(\left\|\Delta_{k, n}\right\|^{3}\right)\right] .
\end{aligned}
$$

Therefore we have

$$
\begin{aligned}
& \left\|\Delta_{j+1, n}-\phi^{*}\left(\Delta_{j+1, n}\right) \phi\right\| \\
& \quad \leqq A \alpha^{j}\left\|\Delta_{0, n}\right\|+\frac{A}{n} \sum_{k=0}^{j} \alpha^{j-k}\left[\left\|\Delta_{k, n}\right\|^{2} K(1)+\frac{1}{n} O\left(\left\|\Delta_{k, m}\right\|^{3}\right)\right] .
\end{aligned}
$$

Since $\alpha<1$, we have, from (4.48) and $\left\|\Delta_{0, n}\right\| \leqq R\|p\|$,

$$
\left\|\Delta_{j+1, n}-\phi^{*}\left(\Delta_{j+1, n}\right) \phi\right\| \leqq A \alpha_{j} R\|p\|+\frac{A B^{2}\left[K(\mathbf{l})+\frac{B}{n}\right]}{n} \frac{1-\alpha^{j+1}}{1-\alpha} .
$$

This shows that, for all $0 \leqq j \leqq n$,

$$
\lim _{\rightarrow \infty}\left\|\Delta_{j, n}-\phi^{*}\left(\Delta_{j, n}\right) \phi\right\|=0 \text {. }
$$

Or, since $\phi^{*}\left(\Delta_{j, n}\right)$ is positive and bounded below, by (4.52) we have

and by (4.56)

$$
\lim _{n \rightarrow \infty} \frac{\Delta \cdot \cdot n}{\phi^{*}\left(\frac{n}{\left.\Delta_{j n}\right)}\right.}=\phi, \quad \phi^{*}(\phi)=1, \quad \text { all } 0 \leqq j \leqq n,
$$

where $\left\|\alpha^{-j} O\left(\alpha^{j}\right)\right\|$ and $\|n O(1 / n)\|$ are bounded independently of $j$ and $n$.

To determine the behavior of $\phi^{*}\left(\Delta_{n, n}\right)$, we apply $\phi^{*}$ to (3.5) with $s=F_{J}\left(s_{n}\right)$. We then get

$$
\phi^{*}\left(\Delta_{j+1, n}\right)=\phi^{*}\left(\Delta_{j, n}\right)-\frac{1}{n} \phi^{*}\left[K\left(\Delta_{j, i}\right)+O\left(\frac{1}{n}\right)\right],
$$

where $O(1 / n)=(1 / n)\left(O\left\|\Delta_{j, n}\right\|^{3}\right)$ and, by (4.48), satisfies

(4.61) $\|n O(1 / n)\| \leqq D<\infty$, with $D$ a constant independent of $n, j$, and $\lambda$ in $\Lambda$.

By (4.52), $\phi^{*}\left(\Delta_{n+1, n}\right)>0$ for $n$ sufficiently large, independent of $j$. Therefore we can consider the identity

$$
\frac{1}{\phi^{*}\left(\Delta_{j+1, n}\right)} \equiv \frac{1}{\phi^{*}\left(\Delta_{j, n}\right)}+\frac{1}{\phi^{*}\left(\Delta_{j, n}\right)}\left[1-\frac{\phi^{*}\left(\Delta_{j+1, n}\right)}{\phi^{*}\left(\Delta_{j, n}\right)}\right] \frac{\phi^{*}\left(\Delta_{j, n}\right)}{\phi^{*}\left(\Delta_{j+1, n}\right)} .
$$


For brevity, we denote the last term in this identity by $A_{j, n}$. Using the homogeneous and quadratic nature of $K$, we compute $A_{j, n}$ from (4.60) as

$$
A_{j,}=\frac{1}{n} \phi^{*}\left[K\left(\frac{\Delta_{j, n}}{\phi^{*}\left(\Delta_{j, n}\right)}\right)+\frac{O(1 / n)}{\left[\phi^{*}\left(\Delta_{j, n}\right)\right]^{2}}\right]\left(1+O\left(\frac{1}{n}\right)\right) .
$$

Or, using (4.59),

$$
A_{j, n}=\frac{1}{n} \phi^{*}\left(K\left[\phi+O\left(\alpha^{j}\right)+O\left(\frac{1}{n}\right)\right]\right)+O\left(\frac{1}{n}\right)^{2} .
$$

Now $K$ is a continuous quadratic polynomial, and hence

$$
K\left[\phi+O\left(\alpha^{j}\right)+O\left(\frac{1}{n}\right)\right]=K\left[\phi+O\left(\frac{1}{n}\right)\right]+O\left(\alpha^{j}\right) .
$$

If we now sum (4.62) on $j$ from 0 to $n-1$ and divide by $n$, we obtain by the two previous equations

$$
\begin{aligned}
\frac{1}{n \phi^{*}\left(\Delta_{n, n}\right)}= & \frac{1}{n \phi^{*}\left(\Delta_{0, n}\right)}+\frac{1}{n} \sum_{j=0}^{n-1} A_{j}, \\
= & \frac{1}{\phi^{*}(\lambda p)+O\left(\frac{1}{n}\right)}+\phi^{*}\left(K\left[\phi+O\left(\frac{1}{n}\right)\right]\right) \\
& +\frac{1}{n}\left[O\left(\frac{1-\alpha^{n}}{1-\alpha}\right)+O(\ln n)\right] .
\end{aligned}
$$

Therefore, since $K$ is continuous,

$$
\lim _{n \rightarrow \infty} n \phi^{*}\left(\Delta_{n,: 1}\right)=\frac{\phi^{*}(\lambda p)}{1+\phi^{*}(\lambda p) \phi^{*}[K(\phi)]}, \lambda p=\sum_{i=1}^{k} \lambda_{i} p_{i} .
$$

In combination with (4.58) for $j=n$, this gives (4.43) for $\lambda$ in the set (4.49) and hence in all of $\Lambda$. Since any $\lambda=\left(\lambda_{1}, \cdots, \lambda_{k}\right)$ with $\operatorname{Re}(. ?)>0$ is contained in some such compact set $\Lambda$, we have completed the proof.

5. Limiting distributions. We have interpreted $F_{n}(x, s), x$ in $X$, as defining a probability generating functional for a random variable $Z_{n}$ with range in the set of functionals in $C^{*}(X, \Sigma)$ defined by additive, nonnegative and integer-valued set functions on $\Sigma$. In certain cases, this is defined for each $n \geqq 1$ and $x$ in $X$, by a measure on the range of $Z_{n}$ [11]. If $X$ is partitioned into disjoint sets $A_{i}$, $\bigcup^{k}=1 A_{i}=X$, and $s=\sum_{i=1}^{k} \lambda_{i} \chi_{1 i},\left|\lambda_{i}\right| \leqq 1$, then $F_{n}(x, s)$ is a generating function in the $\lambda_{i}$. The coefficient or $\lambda_{1}^{r_{1}} \cdots \lambda_{k}^{r_{k}}$ in a series expansion of $F_{n}(x, s)$ is the probability that $Z_{n}\left(A_{i}\right)=r_{i}, i=1, \cdots, k$, given that $Z_{0}=e_{x}$. In particular, $F_{n}(x, 0)$ is the probability that $Z_{n}$ is the zero functional, given that $Z_{0}=e_{x}$.

With the independence condition (2.24) we have

$$
\operatorname{Pr}\left(Z_{n}=0 \mid Z_{0}=\sum_{i=1}^{k} r_{i} e_{x_{i}}\right)=\prod_{i=1}^{k}\left[F_{n}\left(x_{i}, 0\right)\right]^{r_{i}} \text {. }
$$


From Theorem 4 we have

$$
\lim _{n \rightarrow \infty} \operatorname{Pr}\left(Z_{n}=0 \mid Z_{0}=\sum_{i=1}^{k} r_{i} e_{x_{i}}\right)=1,
$$

and the process tends to die.

We are interested in $Z_{n}$ with the condition that $Z_{n} \neq 0$. Since finite linear combinations of characteristic functions are dense in $C(X, \Sigma)$, it follows that for given $Z_{0}=\sum_{i=1}^{k} r_{i} e_{x_{i}}$ and $h$ in $C(X, \Sigma), Z_{n}(h)$ is a complex-valued random variable. We study $Z_{n}$ by investigating its values $Z_{n}(h)$ on $C(X, \Sigma)$.

THEOREM 8. For a CPR process the expected value of $Z_{n}(h), h$ in $C(X, \Sigma)$, given that $Z_{n} \neq 0$, satisfies

$$
\lim _{n \rightarrow \infty} \mathscr{E}\left(\frac{Z_{n}(h)}{n} \mid Z_{n} \neq 0\right)=\phi^{*}[K(\phi)] \phi^{*}(h)
$$

for all $Z_{0}$ of the form $Z_{0}=\sum_{i=1}^{k} r_{i} e_{x_{i}}$, where $M \phi=\phi, M^{*} \phi^{*}=\phi^{*}, \phi^{*}(\phi)=1$.

Proof. For a given $Z_{0}$, the generating functional for $Z_{n}$, given that $Z_{n} \neq 0$, is

$$
\frac{\prod_{i=1}^{k}\left[F_{n}\left(x_{i}, s\right)\right]^{r_{i}}-\prod_{i=1}^{k}\left[F_{n}\left(x_{i}, 0\right)\right]^{r_{i}}}{1-\prod_{i=1}^{k}\left[F_{n}\left(x_{i}, 0\right)\right]^{r_{i}}} .
$$

To compute (5.3), we take the variation of (5.4) with differential $h$ and let $s$ tend to 1 . This gives

$$
\mathscr{E}\left(Z_{n}(h) \mid Z_{0} \neq 0\right)=\frac{\sum_{i=1}^{k} r_{i} M^{n}(h)\left(x_{i}\right)}{1-\prod_{i=1}^{k}\left[F_{n}\left(x_{i}, 0\right)\right]^{r_{i}}} .
$$

The numerator in (5.5) is just $Z_{0}\left(M^{n}(h)\right)$ and approaches $Z_{0}(\phi) \phi^{*}(h)$ as $n \rightarrow \infty$. Dividing (5.5) by $n$ gives

$$
\lim _{n \rightarrow \infty} \mathscr{E}\left(\frac{Z_{n}(h)}{n} \mid Z_{0} \neq 0\right)=\frac{Z_{0}(\phi) \phi^{*}(h)}{\lim _{n \rightarrow \infty} n\left(1-\prod_{i=1}^{k}\left[F_{n}\left(x_{i}, 0\right)\right]^{r i}\right)} .
$$

From Theorem 6 we have

$$
F_{n}\left(x_{i}, 0\right)=1-\frac{1}{\phi^{*}[K(\phi)]} \frac{\phi\left(x_{i}\right)}{n}+O\left(\frac{1}{n}\right),
$$

where $n o(1 / n) \rightarrow 0$ as $n \rightarrow \infty$. Substituting from (5.7) in the denominator of (5.6), we get 


$$
\lim _{n \rightarrow \infty} \mathscr{E}\left(\frac{Z_{n}(h)}{n} \mid Z_{n} \neq 0\right)=\frac{Z_{0}(\phi) \phi^{*}(h) \phi^{*}[K(\phi)]}{\sum_{i=1}^{k} r_{i} \phi\left(x_{i}\right)} .
$$

Since $\sum_{i=1}^{k} r_{i} \phi\left(x_{i}\right)=Z_{0}(\phi)$, this gives (5.3) and completes the proof.

This theorem suggests that $Z_{n} / n$, given that $Z_{n} \neq 0$, tends to a random scalar multiple of the given linear functional $\phi^{*}$, independent of the initial state $Z_{0}$. If the process is alive at the $n$th generation, it tends to have a population size proportional to $n$ with the distribution among types being determined by the value of $\phi^{*}$ on characteristic functions.

We investigate this more thoroughly by considering the conditional distributions for the nonnegative, vector-valued, random variables, defined for $p$ in $C(X, \Sigma), p \geqq 0$, and for disjoint sets $A_{i}, \bigcup_{i=1}^{k} A_{i}=X$, by

$$
W_{n}(p)=\left(\frac{\left(Z_{n}\left(p_{1}\right), \cdots, Z_{n}\left(p_{k}\right)\right)}{n \phi^{*}[K(\phi)]} \mid Z_{n} \neq 0\right), \quad p_{i}=p \chi_{A_{i} \cdot} .
$$

THEOREM 9. For a CPR process, it follows that if $p_{i}=p \chi_{A_{i}}, \bigcup_{i=1}^{k} A_{i}=X$, $p \geqq 0$ in $C(X, \Sigma)$, then for real constants $\alpha_{1}, \alpha_{2}, \cdots, \alpha_{k}$

$$
\begin{aligned}
& \lim _{n \rightarrow \infty} \operatorname{Pr}\left(\frac{Z_{n}\left(p_{i}\right)}{n \phi^{*}\left[\frac{K(\phi)]}{K()}\right.} \leqq\right. \\
&= \begin{cases}0 & \text { if some } \left.\alpha_{i}<0, i=1, \cdots, k \mid Z_{n} \neq 0\right) \\
1-\exp \left(-\sqrt{k} \min _{i}\left[\frac{\alpha_{i}}{\phi^{*}\left(p_{i}\right)}\right]\right) & \text { if all } \alpha_{i} \geqq 0,\end{cases}
\end{aligned}
$$

for any $Z_{0}$ of the form $Z_{0}=\sum_{i=1}^{m} r_{i} e_{x_{i}}, r_{i}$ positive integers.

Proof. This result is trivial for $p=0$. For $p \geqq 0, p \neq 0$, we compute the $k$ dimensional Laplace transform of the distribution of the nonnegative, vectorvalued random variables $W_{n}(p)$ defined by (5.9).

For $Z_{0}=\sum_{i=1}^{m} r_{i} e_{x_{i}}$, the continuity of $F_{n}$ and the density of finite linear combinations of characteristic functions in $C(X, \Sigma)$ leads to

$$
\mathscr{E}\left(e^{-W_{n}(\lambda p)}\right)=\frac{\prod_{i=1}^{m}\left[F_{n}\left(x_{i}, s_{n}\right)\right]^{r_{i}}-\prod_{i=1}^{m}\left[F_{n}\left(x_{i}, 0\right)\right]^{r_{i}}}{1-\prod_{i=1}^{m}\left[F_{n}\left(x_{i}, 0\right)\right]^{r_{i}}}
$$

with $s=\exp \left[-\lambda p / n \phi^{*}[K(\phi)]\right], \lambda p=\sum_{i=1}^{k} \lambda_{i} p_{i}$, and $\operatorname{Re}\left(\lambda_{i}\right)>0$. With $G_{n}$ defined by (4.40), this can be written as

$$
\mathscr{E}\left(e^{-W_{n}(\lambda p)}\right)=1-\frac{1-\prod_{i=1}^{m}\left[1-\frac{G_{n}\left(x_{i}, p, \lambda\right)}{n}\right]^{r_{i}}}{1-\prod_{=1}^{m}\left[F_{n}\left(x_{i}, 0\right)\right]^{r_{i}}} .
$$


From Theorem 6 and Theorem 7 , with $\lambda p$ replaced by $\lambda p / \phi^{*}[K(\phi)]$, we have after expanding the products in (5.12)

or

$$
\lim _{n \rightarrow \infty} \mathscr{E}\left(e^{-W_{n}(\lambda p)}\right)=1-\frac{\sum_{i=1}^{k} \lambda_{i} \phi^{*}\left(p_{i}\right) Z_{0}(\phi)}{\left[1+\sum_{i=1}^{k} \lambda_{\imath} \phi^{*}\left(p_{i}\right)\right] \phi^{*}[K(\phi)]} \frac{\phi^{*}[K(\phi)]}{Z_{0}(\phi)},
$$

$$
\lim _{n \rightarrow \infty} \mathscr{E}\left(e^{-W_{n}\left(\lambda_{i}\right)}\right)=\frac{1}{1+\sum_{i=1}^{k} \lambda_{i} \phi^{*}\left(p_{i}\right)}, \quad \operatorname{Re}\left(\lambda_{i}\right)>0 .
$$

The last term in (5.14) is the $k$-dimensional Laplace transform of the exponential distribution given in (5.10). This is most easily seen by realizing that this exponential distribution puts unit mass on the ray $\alpha\left(\phi^{*}\left(p_{1}\right), \cdots, \phi^{*}\left(p_{k}\right)\right), \alpha \geqq 0$, with a distribution $1-e^{-x}$. Since (4.14) holds for all $\operatorname{Re}(\lambda)>0$, it follows easily from standard theorems on Fourier transforms of probability distributions $[4$, p. 102] thac $(5.10)$ is true. The proof is thus complete.

For the finite dimensional subspace $C_{k}$ of $C(X, \Sigma)$ having as basis the functions $p \chi_{A_{1}}, A_{i}$ disjoint and $\bigcup_{i=1}^{k} A_{i}=X$, and $p \geqq 0$ in $C(X, \Sigma), Z_{n}$ defines a random positive vector in the dual space to $C_{k}$. This last theorem shows that $Z_{n} / n \phi^{*}[K(\phi)]$, given $Z_{n} \neq 0$, has a distribution in $C_{k}^{*}$ which converges with $n \rightarrow \infty$, to a random scalar multiple of the given functional $\left(\phi^{*}\left(\chi_{A_{1}}\right), \cdots, \phi^{*}\left(\chi_{A_{k}}\right)\right.$ ), expressed relative to a basis in $C_{k}^{*}$ dual to that in $C_{k}$.

\section{Applications.}

I. FINITE TYPES. Let $X$ be a finite set and $\Sigma$ the set of all subsets of $X$. Then $C(X, \Sigma)$ is simply a $k$-dimensional complex vector space, and $F$ is defined by $k$ generating functions of $k$ complex variables. The range of $Z_{n}$ is the set of nonnegative $k$-vectors with integer components, a subset of $C^{*}(X, \Sigma)$. Theorem 9 characterizes the limiting distribution of $Z_{n} / n$, given $Z_{n} \neq 0$, as $n \rightarrow \infty$.

II. A NUCLEAR REACTOR MODEL. We consider a homogeneous sphere of fissionable material. We assume that the velocity of neutrons in this sphere is constant and that motion is interrupted only by a collision with a nucleus. Upon collision, a random number of neutrons is produced with an isotropic angular distribution. The collision process is described by a probability generating function:

$$
f(\lambda)=\sum_{n=0}^{\infty} a_{n} \lambda^{n}
$$

where $\lambda$ is a complex parameter and $a_{n}$ is the constant probability that $n$ neutrons are produced.

A branching process is defined in which a neutron is typed according to the spherical shell in which it was produced by fission $[11 ; 12 ; 18]$. The random variable $Z_{n}$ is defined by the number of neutrons which can be traced 
back to a trigger neutron through $n$ fissions. The discrete time of this branching process is related in a complicated manner to physical time.

We parametrize the spherical shells by a real variable $x, 0 \leqq x \leqq R<\infty$, where $R$ is the radius of the reactor. Then the set of types $X$ is the interval $[0, R]$, and we take the $\sigma$-field of Borel sets as the set $\Sigma$.

With the assumption of homogeneity and isotropy, a neutron born at $s$ will have a first collision in the spherical shell between $y$ and $y+\Delta y$ with probability $K(x, y) \Delta y$, where (see [5])

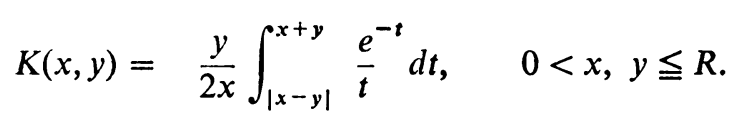

The unit of length has been chosen as a mean free path.

The generating functional of our process is

$$
F(x, s)=1-\int_{0}^{R} K(x, y)[1-f(s(y))] d y,
$$

where $s$ is any Borel-measurable function in $C(X, \Sigma)$ with $|s(x)| \leqq 1$ for all $x$ in $X$. The term $1-\int_{0}^{R} K(x, y) d y$ in (6.3) measures the probability of escape without collision.

Since $f$ is a continuous function it is Borel measurable, and hence $f(s)$ is a Borel-measurable function on $X$ for measurable $s$, and so is $F(s)$. It follows easily then that conditions (2.14) are satisfied by $F$.

The expectation operator $M$ is given by

$$
M(h)(x)=f^{\prime}(1) \int_{0}^{R} K(x, y) h(y) d y .
$$

The operator $M$ can be derived from an operator defined for functions on threedimensional real space, and the results of $[20$, p. 29] show that the third iterate of $M$ is defined by a continuous kernel. It follows readily that this is a compact operator in $C(X, \Sigma)$. (See [6, p. 260].) Therefore (2.30) is satisfied. By the Lebesgue Dominated Convergence Theorem [6, p. 151] it follows that (2.29) is satisfied. So $F$ satisfied the conditions imposed in $\S 2$ as long as $f$ is not linear and its first three derivatives have limits at $\lambda=1$.

For a given value of $f^{\prime}(1)>1$, there is a unique $R$ for which $\sigma(M)=1$. (See [18].) A good approximation to $R$ is given [19] by solving for $R$ in

$$
\begin{aligned}
\frac{1}{f^{\prime}(1)} & =1-\sin ^{2} \gamma+R\left[\ln \left(\frac{\pi}{\gamma}\right)-C i(2 \pi)+C i(2 \gamma)\right], \\
\gamma \tan \left(\gamma-\frac{\pi}{2}\right) & =R, \quad \frac{\pi}{2} \leqq \gamma \leqq \pi .
\end{aligned}
$$


Good approximations to $\phi$ and $\phi^{*}$ are given [19] by

$$
\phi=\frac{\sin \left(\frac{\gamma x}{R}\right)}{x}, \phi^{*}(f)=c \int_{0}^{R} x \sin \left(\frac{\gamma x}{R}\right) f(x) d x,
$$

with

$$
c=\frac{2 \sqrt{ }\left(R^{2}+\gamma^{2}\right)}{R\left(R+\sqrt{\left.\left(R^{2}+\gamma^{2}\right)\right)}\right.} .
$$

Theorem 8 shows that any critical reactor which is described by this mathematical model will produce a finite number of neutrons when initated by a finite number of trigger neutrons.

Fluctuations in the number produced are measured by Theorem 9. For example, let $A$ be any Borel set in $[0, R]$ with nonzero Lebesgue measure. Then $\phi^{*}\left(\chi_{A}\right)>0$ determines the importance of this set to the branching process. The probability $P_{n}(A, \alpha)$ that in the $n$th generation the number of neutrons produced in the spherical shells defined by $A$ exceeds $\alpha n\left(f^{\prime \prime}(1) / 2 f^{\prime}(1)\right) \phi^{*}\left(\phi^{2}\right) \phi^{*}\left(\chi_{A}\right)$, given that this number is not 0 , satisfies

$$
\lim _{n \rightarrow \infty} P_{n}(A, \alpha)=e^{-x}, \quad 0 \leqq \alpha<\infty .
$$

Here we have used the explicit expression for $K$, i.e.,

$$
K(h)=f^{\prime \prime}(1) \int_{0}^{R} K(x, y) h^{2}(y) d y .
$$

The results of this paper will apply to more complicated reactor models. In a trivial manner, the assumption of homogeneity can be replaced by the assumption that the reactor is stratified into spherical shells. This will simply result in $f$ of (6.1) being dependent upon the variable $x$, after a proper scaling of $x$ by the total cross section. Of course, the characterization of criticality and of the eigenfunctions $\phi$ and $\phi^{*}$ will be different. (See [19] for a treatment of core and reflector.)

A problem of considerable interest and difficulty is that of relating the characterization of neutron population by generation to its characterization in real time.

\section{REFERENCES}

1. S. Bochner and W. T. Martin, Several complex variables, Princeton Univ. Press, Princeton, N. J., 1948.

2. V. P. Čistyakov, Transitional phenomena in branching processes with n-types of particles, Teor. Veroyatnost. i Primenen. Vol. 6, No. 1, (1961), 31-45. (Russian)

3. H. E. Conner, A limit theorem for a position-dependent branching process, J. Math. Anal. Appl. 3 (1961), 560-591. 
4. H. Cramer, Mathematical methods of statistics, Princeton Univ. Press, Princeton, N. J., 1946.

5. B. Davison, Neutron transport theory, Oxford Univ. Press, New York, 1957.

6. N. Dunford and J. Schwartz, Linear operators. Part I, General theory, Interscience, New York, 1959.

7. C. J. Everett and S. Ulam, Multiplicative systems in several variables. I, II and III, Los Alamos Scientific Laboratory Declassified Documents, LA-683, 690, 707, 1948.

8. P. Fatou, Sur les équations fonctionelles, Bull. Soc. Math. France 47 (1919), 161-271.

9. P. Halmos, Measure theory, Van Nostrand, New York, 1950.

10. T. E. Harris, Some mathematical models for branching processes, Proceedings of the Second Berkeley Symposium, Univ. California Press, Berkeley, Calif., 1951, pp. 305-328.

11. - The theory of branching processes, Springer, Berlin (to appear).

12. - On one-dimensional neutron multiplication, The RAND Corporation, Research Memorandum RM-2317 (ASTIA No. AD 233143), Santa Monica, Calif., 1960.

13. E. Hille and R. S. Phillips, Functional analysis and semi-groups, Amer. Math. Soc. Colloq. Publ., Vol. 31, Amer. Math. Soc. ,Providence, R. I., 1957 (revised edition).

14. M. Jirina, The asymptotic behaviour of branching stochastic processes, Czechoslovak Math. J. 7(82) (1957), 130-153. (Russian)

15. S. Karlin, Positive operators, J. Math. Mech. 8 (1959), 907-937.

16. M. G. Krein and M. A. Rutman, Linear operators leaving invariant a cone in a Banach space, Amer. Math. Soc. Transl. No. 26, (1951).

17. J. E. Moyal, Multiplicative population chains, Acta Math. (to appear).

18. T. W. Mullikin, Neutron branching processes, J. Math. Anal. Appl. 3 (1961), 507-525.

19. - Estimates of critical dimensions of spherical and slab reactors, J. Math. Anal. Appl. 5 (1962), 184-199.

20. I. G. Petrovskii, Integral equations, Graylock, Rochester, New York, 1957, (translation).

21. B. A. Sevast'yanov, On the theory of branching random processes, Reports of the Academy of Sciences of SSSR, Vol. 59, 1948, 1407-1410. (Russian)

22. - The theory of branching random processes, Uspehi Mat. Nauk. Vol. 6, No. 6, (1951), 47-99. (Russian)

23. - Transitional phenomena in branching stochastic processes, Teor. Veroyatnost. i Primenen, Vol. 4, No. 2, (1959), 121-135. (Russian)

24. A. M. Yaglom, Certain limit theorems of the theory of branching random processes, Reports of the Academy of Sciences of USSR, Vol. 56, No. 8, 1947, 795-798. (Russian)

THE RAND CORPORATION, SANTA Monica, California 